\title{
A LEGACY SYSTEMS USE CASE RECOVERY METHOD
}

\author{
Philippe Dugerdil, Sebastien Jossi \\ HEG, Univ. of Applied Sciences of Western Switzerland, 7 route de Drize CH-1227 Geneva, Switzerland \\ philippe.dugerdil@hesge.ch
}

Keywords: Reverse specification, dynamic analysis, static analysis, execution trace, branch condition analysis.

\begin{abstract}
During the development of a legacy system reverse engineering method we developed a technique to help with the recovery of the system's use-cases. In fact, our reverse-engineering method starts with the redocumentation of the system's use-case by observing its actual users. But these use-cases are never complete and accurate. In particular, the many alternative flows are often overlooked by the users. This paper presents our use-case recovery methodology as well as the techniques we implemented to identify all the flows of the legacy system's use-case. Starting from an initial use-case based on the observation of the users, we gather the corresponding execution trace by running the system according to this use-case. The analysis of this execution trace coupled with a static analysis of the source code lets us find the possible alternative execution paths of the system. The execution conditions for these paths are analyzed to establish the link to the use-case level. This lets us synthesize alternative flows for the use-case. Next we run the system again following these alternative flows to uncover possible new alternative paths, until one converges to a stable use-case model.
\end{abstract}

\section{INTRODUCTION}

Generally, legacy systems documentation is at best obsolete and at worse non-existent. Often, the developers are not available anymore to provide the maintainers with information on these systems. In such situations the only people that still have a good perspective on the system are its users. In fact they are usually well aware of the business context and business relevance of the programs. In short, their interactions with the system represent instances of relevant use-case.

The iterative and incremental reverse-engineering technique we developed starts from the recovery of the use-cases of the system. Then, by incrementally rebuilding the analysis models, we are able to recreate the traceability links between the business functions and the source code of the system. In summary, this reverse-engineering method works through the following steps:

1. Re-document the system use-cases;

2. Design the Unified Process' robustness (analysis) diagrams associated to these use-cases (Jacobson et al. 1999);

3. Execute the system according to the use-cases and record of the execution trace;

4. Analyze the execution trace and identify the classes involved in the trace;
5. Map the classes in the trace to the stereotypes of the robustness diagram.

6. Re-document the architecture of the system by clustering the classes based on their role in the implementation of the use-case.

The efficiency of this method has successfully been tested on 2 large systems (Dugerdil\&Jossi 2008, Dugerdil\&Jossi 2007). Since our approach rests fundamentally on the recovered use-cases, their quality and completeness are fundamental to the performance of our reverse-engineering method. However as we rely on system users to recover the use-cases, the latter are never complete and accurate especially regarding the alternative flows. Therefore we developed a technique to recover complete usecases from the rough one given by the users.

The topic of this paper is to present our use-case recovery approach. In contrast with other published use-case recovering techniques based on the analysis of the source code only (see for example ( $\mathrm{Li}$ et al. 2007)) our approach rests on a first "draft" of the use-case provided by users. This version is later completed by analyzing the behavior of the program as well as the source code of the classes involved in the implementation of the use-case.

This position paper presents work in progress. It is structured as follows. Section 2 discusses the problem of recovering meaningful, i.e. relevant, use case in a given domain. Section 3 presents the use- 
case recovery process and section 4 gives some hints on the implementation technique. Section 5 discusses the related work. Section 6 concludes that paper and presents future work.

\section{RECOVERING RELEVANT USE-CASES}

According to (Leffingwell\&Widrig 2003) “A use case describes sequences of actions a system performs that yield an observable result of value to a particular actor". Furthermore, "It (the use-case) focuses on the value that the customer wants from the system, not on how we subdivide and structure the functionality within the system" (Bittner 2001). Therefore, when trying to reverse-engineer the usecases from a legacy system, the goal is not to generate any arbitrary set of statements, but to actually recover a relevant one. Since software specification lies at the boundary between business and engineering (i.e. expressing functional requests based on business justifications), the recovered usecases must be relevant to, and consistent with, the business tasks of the users. However in the vast majority of situation, if not all, the software source code does not contain any substantial structured business information to justify the software in business terms. In other words the business relevance of a given piece of code is not to be found in the source code itself but lies outside the code (in the head of the software analyst). This situation bears some similarity to the linguistic domain where it is well known that the understanding of a text requires pre-existing knowledge of the domain (Roche 2006). Therefore, it is clear that any use-case recovery technique that is based on the analysis of the source code only is bound to fail. In particular the structure of the use-case (business level) is very loosely linked to the structure of the software (technical level) implementing it. Of course, both structures are not completely uncorrelated, but the correlation is much too weak to link the technical structure to the usecase structure. Again, this is because the software engineering and technical principles that lead to the program structure are orthogonal to the business requirements. In particular, the structure of the code is driven by quality attributes considerations like maintainability or performance (Bass et al. 2003).

However, since the actual users of the system have a good perspective on the business relevance of the system, they are able to execute scenarios that are relevant to the business. But we know that the latter are not complete and accurate enough to be considered good use-cases. We will therefore complete them by selectively searching the source code for variants of the scenario and then abstracting the information to generate complete relevant usecases. To illustrate this technique, we call the initial scenario the "backbone" of the use-case, to which we incrementally attach extra information that we obtain from searching the source code.

\section{USE-CASE RECOVERY PROCESS}

First, the legacy system's source code is instrumented to generate an execution trace for any scenario performed on the system. The instrumented source code is then recompiled and installed on the machine. Next, the scenario we recovered from the user is played on the machine and the execution trace is recorded. The latter then contains the sequence of methods or functions executed while performing the scenario. The format of the execution trace is quite classical. Each method call, called an "event", has the following form:

[packageName][className][methodSignature][processId]

After having recorded the execution trace it is analyzed to identify the methods executed. For each method called, we look for conditional statements in its source code. When such a statement is found, the non-executed path represents a candidate for a variant of the use-case. This is illustrated in Fig. 1. The small squares symbolize the program statements and the central broken line represents the sequence of statements executions for the "backbone". The small horizontal arrows represent the search for variants around the "backbone" statements. The purpose is to find the statements that could possibly be called but haven't been. These may represent extra steps in the main flow or steps of the alternative flows.

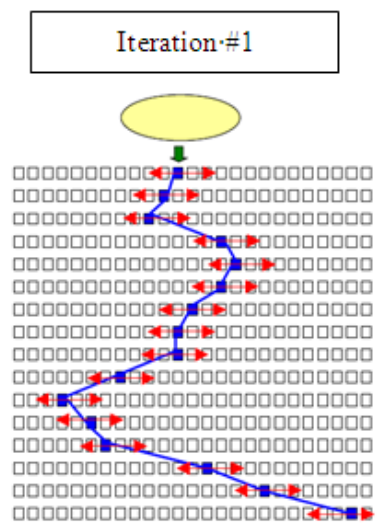

Figure 1. Iteration 1 performed on the "backbone" 
The next task is to link the conditional statements in the code to the user interfaces involved in the "backbone" scenario, to know if the alternative statements could possibly be executed by performing some extra user interactions. In other words, we must answer the following question: could the boolean condition of the branching statement be changed by imputing some specific value or selecting some extra option at the user interface level? If yes, the corresponding user interaction is added as an extra step to the scenario. Then the users are asked to validate the relevance of the new scenario (previous one + new interaction). If it is OK, the new scenario is played and a new execution trace is generated. This process is repeated until we reach a stable set of variants. This incremental process is symbolized in figure 2 .

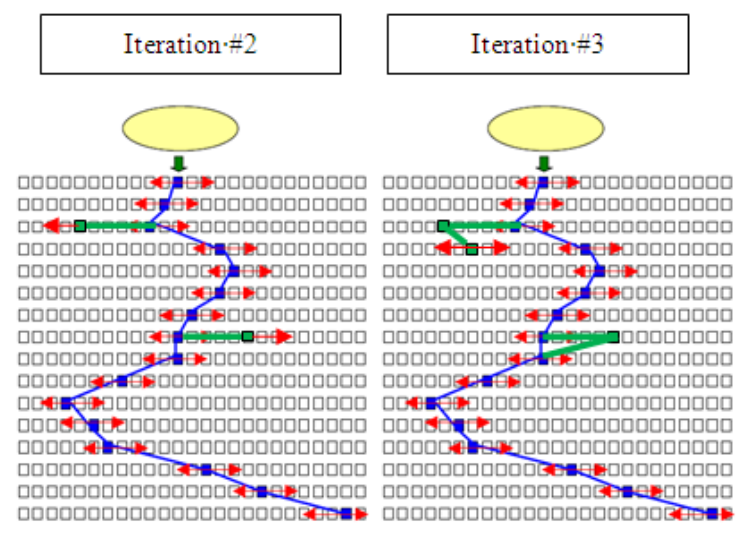

Figure 2. Extra iterations performed on the "backbone"

The new segments of the broken line represent variant of the behavior of the system obtained via the new user interaction determined by the static analysis of the source code. In summary our technique uses dynamic (i.e. execution trace) and static (i.e. source code) analysis.

\section{USE-CASE RECOVERY TECHNIQUE}

The implementation of our approach requires two sources of information:

1. The execution trace of the scenarios;

2. The source code of the classes involved in execution trace.

The whole process is illustrated in figure 3. First, a rough use-case is rebuilt from the scenario recovered from the users. Next the scenario is executed on the instrumented system to get the execution trace. The latter is then recorded and the methods corresponding to each event is analyzed. If a conditional statement is found in that code, this could lead to an alternative behavior (c6 $t()$ in the example) depending on the boolean condition. Then the system tries to link this boolean condition (the $k$ variable in the example) to the user interface of the use-case. This is to check if some user interaction could lead to a change in the boolean value of $\mathrm{k}$. If yes, then the alternative path (c6 $t()$ instead of $\mathrm{C5} t()$ ) will be taken if this action is taken. To identify the non-executed conditional statements in the code, we rest on the analysis of the execution trace again which is represented by the corresponding call tree. Starting from a node that represents an event, we check if the method that could conditionally be executed is a child node of the current node in the class tree. If not, then this represents a potential alternative path and the source code is backward sliced from the condition to uncover alternative scenarios. Backward slicing is a technique to identify all the statements in a program that could possibly influence the value of some variable at a specific step in the program (called the slicing criteria (Binkley\&Gallagher 1996)). Therefore, if a user interaction through a control of the GUI could change the value of a condition in the code, this means that the statements associated to the control must be included in the backward slide from the conditional statement.

In summary the algorithm to find alternatives is the following:

For each event in the trace

Retrieve the conditional statements in its code.

For each conditional statement

If the corresponding method is not a child of the current node

\section{Then}

1. Backward slice the source code of the program from the condition.

2. Analyze the slice to find if there are statements belonging of the scenario's GUI.

3. Check if the GUI statements are associated to some user selectable control.

4. Deduce the value to be inputted/selected through the GUI to change the condition.

$$
\text { endIf }
$$

$$
\text { endFor }
$$

endFor

Technically, the source code of each event (method) is parsed to generate its AST. The latter is then analyzed using the "Visitor » design pattern (Gamma et al. 1995). Both the AST generator and its 

Gesser (Java 1.5 Parser and AST 2010).

In most of the cases, the identification of the method that is called in the trace is easy. However in some cases the calls are ambiguous. parser have been kindly shared by Júlio Vilmar

Let us see a simple example where method1() would be declared in Class1:
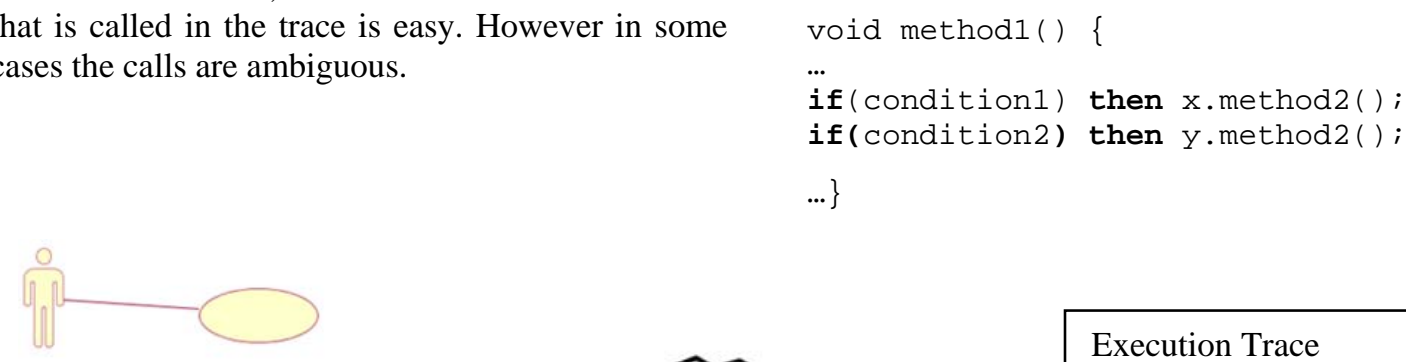

Use Case $\mathrm{X}$ Main flow

1)-

2)--

3)-

4)---------------------
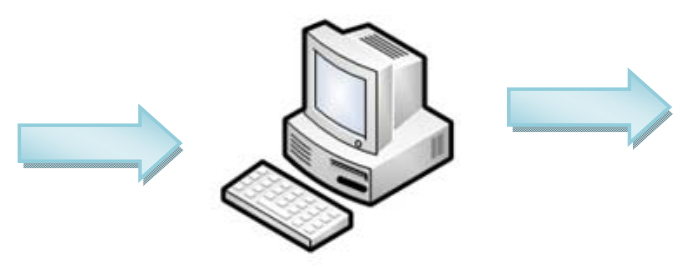

Abstract Syntax Tree (AST)
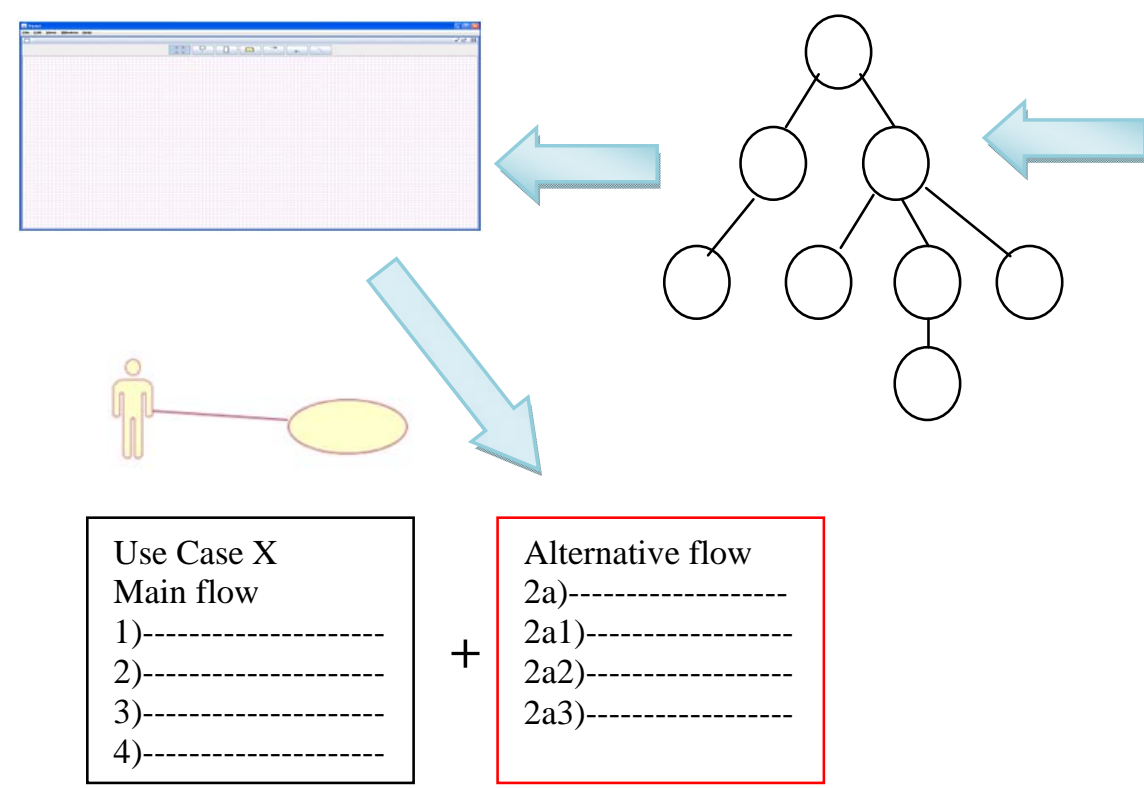

Figure 3. Workflow of our method

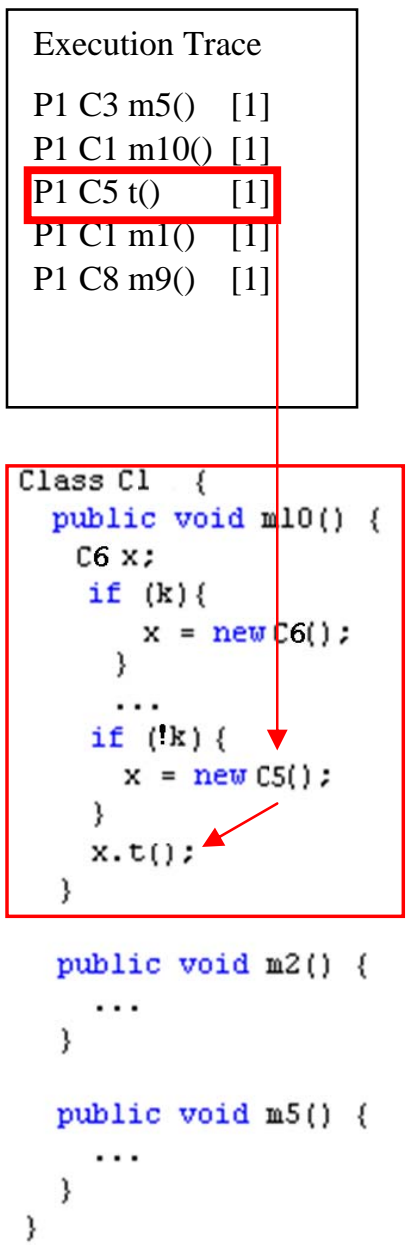

\}
In the execution trace we may get the following sequence of events:

package1 Class1 method1()[1]

We know from the source code of method1( ) that method2() might be called. But what is the exact code executed in this case? In other words was conditon1 or condition2 true? The answer will be known by identifying the class of the object referenced by $\mathrm{x}$ and $\mathrm{y}$. Now the problem comes back to finding these classes. This represents a major issue. The technique is to compute the type of the variable at the location of the conditional statement. Of course, the analysis of the static type (class) of the variable is not enough since the program could assign an instance of a subclass to the variable. We must therefore analyze the assignments statements to the corresponding variables up to the conditional statement. The solution is to launch yet another 
Visitor (Gamma et al. 1995) to identify these assignment statements. While the visitor searches the code a dynamic type table is filled with the conditions that correspond to each of the assignment statements. Figure 4 presents the UML model of such a table. This table helps us to relate the conditions with each other in the code. In fact all the alternative paths in the program are not independent. Many paths will indeed be controlled by the same condition or by a combination of known conditions. The dynamic type table is then used to determine truly independent paths in the program.

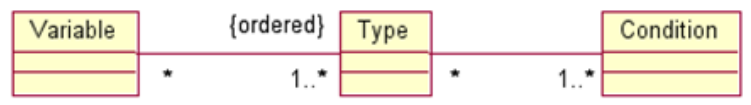

Figure 4. Dynamic type table

When the type of the variables is known, we can disambiguate the call observed in the trace and identify the alternative scenarios. So far we implemented the first part of the method, up to the identification of the conditional statements that could lead to alternatives. The next step will be to backward slice the code to find the alternative flow steps. The conditional statement identification mechanism has been applied on the "FastUML" (FastUML 2010) open source software. First we instrumented its source code and defined a rough use case ("backbone"). Then we played it on the system, got an execution trace and performed our trace analysis technique to find alternative paths. This allowed uncovering 10 alternative calls from the "backbone".

\section{RELATED WORK}

A technique that bears some similarity with ours is the work of (Ko\&Myers 2008). Their debugging application technique is based on dynamic and static analysis and generates a precise call graph by using every invocation found in the source code. This tool allows the user to run buggy functions to uncover the code associated to that function. Moreover, the application dynamically generates questions that the user can ask about program behavior. But this work does not aim at recovering the use-cases of the system. On the topic of reverse specifications, ( $\mathrm{Li}$ at al. 2007) proposed a technique to rebuild a complete use case diagram based on dynamic information (execution trace). They start by retrieving methods that are located at the root of the call trees build from the execution trace. These root methods are supposed to represent the root of the scenarios. But we think this to represent too strong an hypothesis, because it deeply depends on the structure of the code. For instance a root method could implement a menu while the real business function would be located at a much deeper level. In order to rebuild the software behavior model based on execution traces, researchers from the University of Ottawa worked by filtering out utility components to keep only high level elements (Hamou-Lhadj et al. 2005). Their algorithm seems to produce good results, but their technique is not adapted to our problem. Since they are no guided by any business level information, there is no guarantee that the retrieved statement correspond to a relevant use-case. (El-Ramly et al. 2002) have developed a method to recover the use-cases from dynamic information but again they work the other way around by rebuilding the use-case without the guidance of some user-level information. Therefore there is no guarantee that the recovered use-case would be relevant to the users. (Di Lucca\&Fasolino\&De Carlini 2000) also use a dynamic technique to recover the use cases. However the problem here is the very definition of what a use-case means (Leffingwell\& Widrig 2003). In fact, their technique is limited to recording the statements between an input event and the first output event. Therefore this cannot be considered a real use-case since it is limited to analyzing a single feature of the system, not a whole scenario of business value. Finally, (Qin et al.2003) presented a method to retrieve the use-cases of a system by building a branch-reserving call graph. From this graph they could rebuild sequences of user interactions by hand. Although they claim to be able to retrieve plain use-cases, the real question is: what is the business value of the recovered use-cases? Again the key idea is not to retrieve any arbitrary sequence of user interactions but one that represents a real business task. As far as slicing tools are concerned, we explored a few open source tools. (JSlice 2009) seemed at first to be a good candidate. However it cannot work on user defined trace execution format. It must use its own trace format. Therefore we cannot insert it easily in our framework. But the key problem is due to its JVM which is not a standard one but (Kaffee 2009). The latter lacks compatibility with current versions of Java. The (Wisconsin ProgramSlicing Project 2009) released an open source slicing tool. But the latter is designed for $\mathrm{C}$ language only. GrammaTech markets two slicing tools. The first one, (Code Surfer 2009), is actually the commercial version of Wisconsin Program Slicing Project. The second, (Code Sonar 2009), is also designed for C type languages. Finally, the best candidate we found is (Indus 2009)0 developed at Kansas State Univ. It is designed for Java code. This is the one we are concentrating on presently. 


\section{CONCLUSION AND FUTURE WORK}

The fundamental claim of this paper is that it is not possible to recover relevant use-cases of a system by simply analyzing the source code of the system. This is because use-cases represent system usage that must bring a result of business value to the user (Leffingwell\&Widrig 2003) (Bittner 2001). Hence, any set of user interactions with the machine does not represent a use case. It is just a set of user interactions, nothing more. For such a set of interaction to represent a true use-case, all interactions must be targeted at providing the user some result of business value. This business value lies outside of the system. It is in the head of the user (and in some rare cases in the documented business processes involving the IT system). Therefore, our key idea to recover business-relevant use-case is actually to start from an initial user-defined scenario and to incrementally enhance this scenario to converge to a complete use-case. Since we start form a scenario of business value, the value of the use-case resulting from our process is guaranteed. We called the initial scenario the "backbone", since this is a relevant scenario of business value to the user that will later be completed. To perform this completion, we proposed to use dynamic as well as static analysis techniques. The first one let us find the code that is executed while running a scenario (i.e. the execution trace). Then the executed code (the set of events) is searched for alternative execution paths. Once such a path is found, our technique tries to link the condition of its execution to the scenario's GUI. This is to check if some alternative user interaction could possibly lead to the execution of the alternative path. If such a link is found the alternative interaction is presented to the user for validation. If it is validated, the system is run again with the alternative interaction and the corresponding executed code analyzed. This process is repeated until the scenarios converge to a consistent use-case. As of today, the first part of the use-case recovery method is implemented, up to the identification of alternative execution paths. The next step is to use a backward slicing tool to retrieve the corresponding user interactions. This is the work are concentrating on presently. We hope to complete the work by the end of the summer 2010.

\section{ACKNOWLEDGEMENTS}

This work has been done with the support of HESSO Grant $N^{\circ} 24245$ from the Swiss Confederation, which is gratefully acknowledged.

\section{REFERENCES}

Bass L., Clements P., Kazman R. 2003. Software Architecture in Practice, 2nd edition. Adison-Wesley Inc..

Jacobson I., Booch G., Rumbaugh J. 1999. The Unified Software Development Process. Addison-Wesley Professional.

Roche Ch. 2006. How Words Map Concepts . Proc. $10^{\text {th }}$ IEEE EDOCW.

Binkley D.W., Gallagher K.B. 1996. Program Slicing. in: Advances in Computers, vol 43, Academic Press.

Gamma E., Helm R., Johnson R., Vlissides J. 1005. Design Patterns. Elements of Reusable Object Oriented Software. Addison-Wesley Inc.

Ko A., Myers B 2008. Debugging reinvented: asking and answering why and why not questions about program behavior, Proc. IEEE ICSE.

Li Q., Hu S., Chen P., Wu L., Chen W. 2007. Discovering and Mining Use Case Model in Reverse Engineering, Proc. IEEE FSKD.

Hamou-Lhadj A., Braun E., Amyot D., Lethbridge T. 2005. Recovering Behavioral Design Models from Execution Traces. Proc IEEE CSMR.

El-Ramly M., Stroulia E., Sorenson P. 2002. Mining System-User Interaction Traces for Use Case Models. Proc IEEE IWPC.

Di Lucca G. A., Fasolino A. R., De Carlini U. 2000. Recovering Use Case models from Object-Oriented Code: a Threadbased Approach. Proc IEEE WCRE

Qin T., Zhang L., Zhou Z., Hao D., Sun J. 2003. Discovering Use Cases from Source Code using the Branch-Reserving Call Graph. Proc. IEEE APSEC.

Wisconsin Program-Slicing Project. 2009 www.cs.wisc.edu/ wpis/html/

JSlice 2009. jslice.sourceforge.net/

Kaffee 2009 www.kaffe.org/

Indus 2009 indus.projects.cis.ksu.edu/

CodeSonar 2009. www.grammatech.com/products/codesonar/ overview.html

CodeSurfer 2009. www.grammatech.com/products/codesurfer/ overview.html

Leffingwell D, Widrig D. 2003 Managing software requirements, Addison Wesley.

Bittner K. 2001. Why use cases are not "functions" - The Rational Edge.

Dugerdil Ph., Jossi S. 2008. Empirical Assessment of Execution Trace Segmentation in Reverse Engineering. Proc ICSOFT

Dugerdil Ph., Jossi S. 2007 Reverse-Engineering of an Industrial Software Using The Unified Process: An Experiment. Proc IASTED SEA.

Javacc 2010 https://javacc.dev.java.net/

Java 1.5 Parser and AST 2010 javacc.dev.java.net/servlets/ ProjectDocumentView?documentID $=44514 \&$ showInfo $=$ true

FastUML 2010 - sourceforge.net/projects/fastuml/ 